\title{
TOXIC SYMPTOMS IN INFANTS AND CHILDREN WITH GASTRO-INTESTINAL MANIFESTATIONS. *
}

\author{
HERMAN SCHWARZ, M.D., AND JEROME L. KOHN, M.D. \\ NEW YORK
}

Severe toxic symptoms have long been described in infants who are suffering from various intestinal conditions, but the peculiar group of symptoms to be discussed in this article have been brought to the notice of pediatrists very forcibly by Finkelstein and termed by him "alimentary intoxication." Of late, others have grouped these cases under the term "acidosis." Finkelstein's alimentary origin of this condition has not by any means been proven, and as to the acidosis, we have seen many patients who had no diminished carbon dioxid in the blood. This latter fact has been noted by Schloss ${ }^{1}$ and others. We have, therefore, been most careful in the selection of our title, for even the intestinal origin of this condition has not been proven.

The symptom complex is always associated with gastro-intestinal symptoms, and seems to occur almost wholly in the summer months, seen in our hospital wards from July to September, and most frequently during July and August. It does not seem to be much more prevalent during extremely hot summers and does not resemble the cases of heat stroke in infants. Males and females are equally affected. Prematures, or those congenitally weak, seem more prone to this condition than the strong and vigorous, for five out of our series of twenty-three cases were in prematures. It is relatively rare in children when the hygiene and living conditions are good, for we have never seen it occur in the better class of private practice. It is, perhaps, less common in very early infancy, occurring infrequently before the fourth month and most often between the sixth and tenth months. This may be due firstly, to the fact that usually more than one gastro-intestinal upset has occurred before the toxic symptoms supervene, and secondly, that the purely breast fed infant is almost immune from this condition. The age incidence is rather in contradiction to $\mathrm{Ylppo}^{2}$ who seems to think that the new-born has a predilection to acidotic conditions. Yet Seham ${ }^{3}$ was unable to confirm this. In

* Received for publication, Dec. 22. 1920.

* From the Pediatric Service and Pathological Laboratory, Department of Physiological Chemistry, Mt. Sinai Hospital.

1. Schloss, O., and Stetson, R. E.: Am. J. Dis. Child. 13:218 (Feb.) 1917; Schloss, O.: Am. J. Dis. Child. 15:165 (Feb.) 1918.

2. Ylppo, A.: Ztschr. f. Kinderh. 14:268, 1916.

3. Seham, M.: Am. J. Dis. Child. 18:42 (July) 1919. 
our series, all subjects, but one, were in babies artificially fed. This one was an infant, aged 6 weeks, born congenitally weak, and much under weight.

In many instances there was a history of difficult feeding and numerous attacks of diarrhea, yet in some of our cases the history was relatively free from intestinal upsets and the child was doing perfectly well on its formula.

However, in every case gastro-intestinal symptoms were present or had been present before the toxic symptoms occurred. Usually, the child began with an ordinary diarrhea, with a few lumpy and loose movements. This continued either for a few days or a few weeks before vomiting began. At times vomiting and diarrhea began almost simultaneously, the stools becoming gradually more and more frequent and the vomiting persisting. The toxic symptoms usually began a few days or even weeks after the onset of the diarrhea. These were ushered in by restlessness and irritability and followed by apathy, semi-coma and coma.

The children present a picture of being acutely and seriously ill. The skin has an ashen gray, slightly cyanotic appearance, hangs in folds, later frequently becoming scleremic. The fontanel is sunken, upper lids falling over the eyeball, at times resembling a complete ptosis. The cornea is dry and in young infants the iris changes from its characteristic light blue to a brownish gray. ${ }^{*}$ The tongue and the mucous membranes of the mouth have a reddish hue.

The respirations in some cases are apparently normal. In others, they are rapid and shallow, and in still others there is present the typical hyperpnea or deep breathing so frequently described. The rapid respirations may be present without any physical or roentgen signs of pneumonia and hyperpnea present or absent, with or without a low carbon dioxid content of the blood. In some cases there may be a complicating pneumonia, but this is not usual. In our cases it was interesting to note how negative the physical examinations were. The heart rarely presented any adventitious sounds. The electrocardiograph was usually normal showing only an occasional tachycardia. The spleen was rarely palpable and the liver was only moderately enlarged.

The nervous system was markedly affected-irritability, then apathy, finally coma and unconsciousness being the rule. Convulsions were not frequent, and if present they were usually terminal. No abnormal reflexes were noted.

Fever was present in most of the cases. It was very irregular and often very high, extending over many days, although absolutely

4. Schindler, E.: Ztschr. f. Kinderh. 19:153, 1919. 
no physical signs could be elicited in the way of complications referable to the ear, lungs or the pelvis of the kidney. The temperature was not influenced by the withdrawing of food nor was it lowered by the administration of fluids. Many of the terminal temperatures were very high, reaching $106 \mathrm{~F}$. In a few cases observed only a few hours or days, in which the entire history was very short, there was a subnormal temperature.

The vomiting was ustally most persistent and occurred with any form, kind and quantity of food given. The vomiting usually consisted of the ingested food; blood was never seen. Gavage and lavage were of no avail.

The stools were either very watery and frequent, or full of mucus and green; although it was not unusual to have them become almost normal in color and consistency and yet the toxic symptoms continue. Blood was not often present.

The urine was reduced in amount in every case-more than could be accounted for by the diarrhea and vomiting. Even after many subcutaneous and peritoneal injections the quantity excreted was very small. It seemed to us that the quantity of urine excreted was often directly proportionate to the severity of the symptoms, yet there seemed to be no direct relation between the quantity excreted and the blood findings. We hope that during the next summer we shall be able to report more accurately on twenty-four hour quantities in these cases. The specific gravity was usually high and albumin and casts were found in most cases. Sugar (Fehlings), acetone and diacetic acid were present at times, often, however, they were absent, regardless of the amount of voluntary and involuntary starvation and the severity of the symptoms. Excessive amounts of uric acid and ammonium urate crystals ${ }^{5}$ were found in a few cases and are thought by some to signify a bad prognosis. Indican was present in considerable quantity in some cases. The percentage of ethereal sulphate to total sulphates was greatly increased in three cases examined. The kidneys in one case, in which there was almost complete anuria and high retention of nitrogen in the blood, were examined histologically (Dr. Baehr) and found to be practically normal. In this same case the liver was fatty, and there was a marked congestion of the gut.

The duration of this condition varies; at times it is very virulent and lasts but a few days; again, it may last from three to four weeks with periods of marked improvement.

The prognosis is bad; nineteen out of twenty-three patients died ( 87.5 per cent.). This agrees with Schloss, Hamburger ${ }^{6}$ and others.

5. Morgenstern, K.: Ztschr. f. Kinderh. 19:129, 1919.

6. Hamburger, R.: Jahrb. f. Kinderh. 93:25, 1920. 


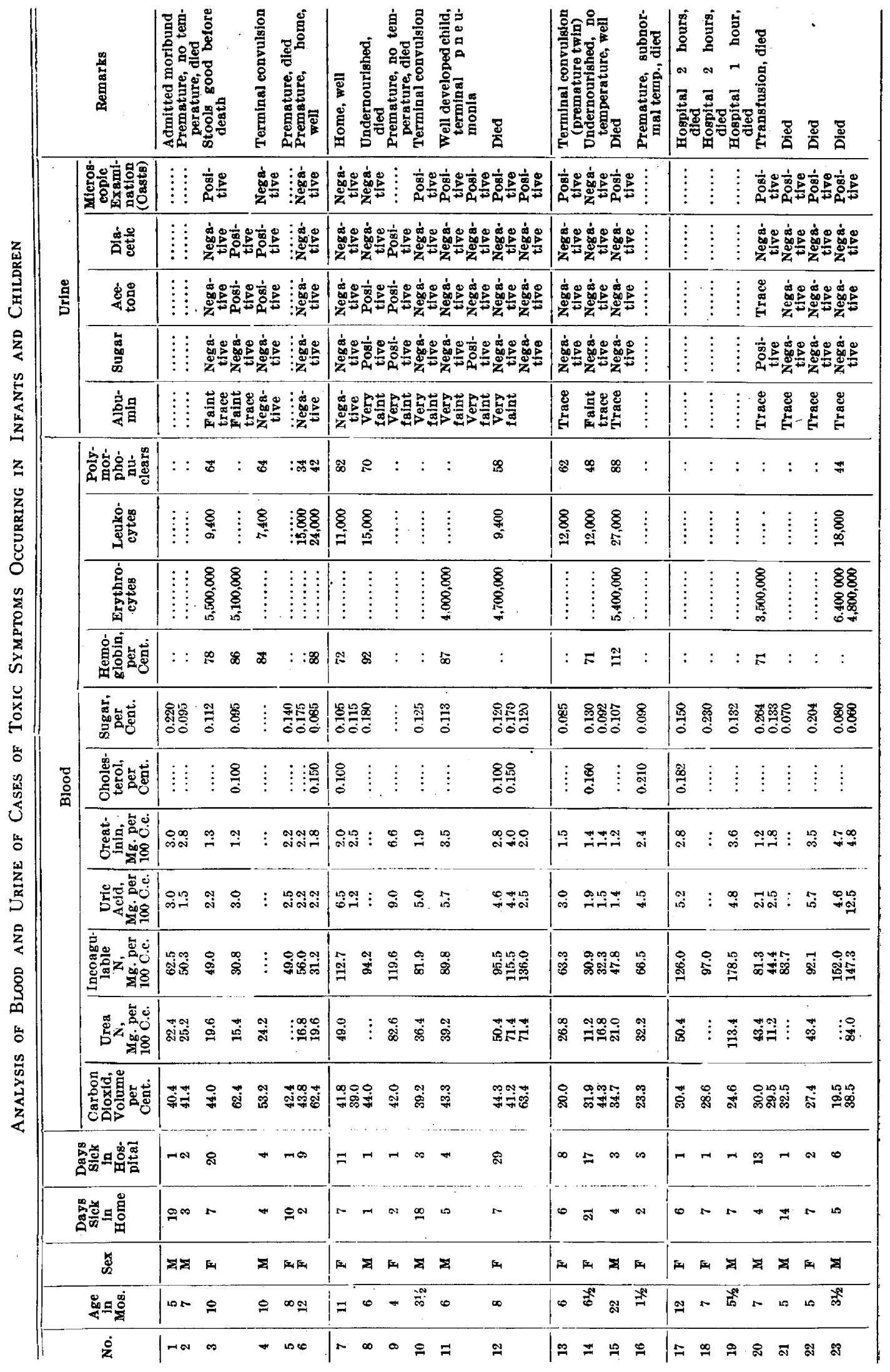


In order to ascertain whether there was any relationship between the clinical symptoms and the blood findings, examinations were made of the urea, nonprotein nitrogen, uric acid, creatinin, carbon dioxid, sugar and cholesterol. ${ }^{7}$ In this way we have investigated twenty-three cases. Unfortunately, because of the grave condition of some of the cases, the length of time under observation and other circumstances, most of the cases had only one blood examination; yet in every case at the time the blood was taken, the children presented the typical symptoms of apathy, coma, vomiting and diarrhea.

Attempting an analysis, it would seem that we could divide our cases into four groups.

In the first group, the blood in regard to nitrogen partitions, cholesterol, sugar, carbon dioxid content was fairly normal. In one of these cases we did a second examination with the same result. These cases presented a clinical picture not differing from that of the others.

A second group of six cases (for convenience called uremic cases) had a normal carbon dioxid content, but the nitrogen constituents were markedly increased. This increase took in all the nitrogen substances which we examined. One of these patients was ill for over a period of weeks, and in this case we had the opportunity of making three examinations, all giving the same results. The urine in this group was not very different from that in the cases with normal blood findings, except that albumin and casts were more frequently found. That this high nitrogen retention was, perhaps, not due to diminished blood volume alone can be inferred from the fact that in some cases the hemoglobin estimations and red blood cell count were not excessively high.

In the third group, consisting of four cases, the only change from the normal was a diminished carbon dioxid content. The blood sugar and nitrogenous elements of the blood were normal. In one of these cases, a second blood examination was taken when the child was recovering. A normal carbon dioxid and other normal figures were obtained.

A fourth group of seven cases was a combination of the second and third groups-a nitrogen retention with a low carbon dioxid content. These cases presented no clinical difference from the others. In two of these cases we were able to make repeated examinations, one subsequently showing slightly less nitrogenous retention.

7. Methods: Urea-urease (Marshall); nonprotein nitrogen-digestion and titration; uric acid and creatinin with Kuttner's modification of Folin's method; sugar-with Epstein's modification of Benedict's method; cholesterol-Bloor's method; carbon dioxid-Van Slyke's gasometric method. 
Schloss also made a number of blood examinations in this type of case and found that the cases could be divided into much the same groups.

In the course of our examination for the uric acid content of the blood, it was noticed that after precipitating the uric acid with silver lactate mixture, the color obtained with the filtrate, using only the phosphotungstate, was exceptionally intense when compared with the filtrate from normal cases. This was noted in some of the cases in which there was no nitrogen retention. Whether this reaction is due to phenol $^{8}$ we are not prepared to say. Further investigation relative to the recent work of Tisdall $^{9}$ with the phosphotungstic-phosphomolybdic reagent and its bearing on phenols, is being pursued.

From the above it would appear that in some cases the blood chemistry is normal; in others there is a diminished carbon dioxid content; in still others only a retention of nitrogenous products and no acidosis, and finally, there is a group with both a nitrogenous retention in the blood and an acidosis. Most likely the underlying factor is the same in all groups.

Finkelstein ${ }^{10}$ and his co-workers have ascribed this toxic symptom complex to the absorption of some of the food principles through a damaged or undamaged gut.

Escherich, ${ }^{11}$ Libman, ${ }^{12}$ Davidson ${ }^{13}$ and many others have tried to ascribe some of these cases as being due to an invasion of a streptococcus or organisms of the dysentery group.

Recently Moro, ${ }^{14}$ Bessau ${ }^{15}$ and their co-workers have shown that there is an invasion of the duodenum by the Bacillus coli in these cases. They believe that these symptoms may be due to the action of these bacteria on the food or to the absorption of products of the bacteria themselves. Pursuing this idea further, Plantenga ${ }^{18}$ in Holland and Hamburger ${ }^{6}$ in Berlin, treated these patients with subcutaneous injections of a polyvalent $B$. coli horse serum with encouraging results.

In our treatment, the main object was to maintain the water balance, the etiology and the processes of this condition being as yet poorly understood. Glucose solution was used partly for nutrition and partly as a diuretic. Sodium bicarbonate solutions were not used, for it

8. Folin, O., and Denis, W.: J. Biol. Chem. 12:239, 1912.

9. Tisdall, F. F.: J. Biol. Chem. 44:409, 1920.

10. Finkelstein, H.: Lehrb. d. Säuglingskrankh. 1911, Part 2, pp. 189-279.

11. Escherich. T.: Jahrb. f. Kinderh. 49:137, 1899. 1897.

12. Libman, E.: Centralbl. f. Bakteriol., Parasitenk. u. Infektionk. 22:376,

13. Davison, W. C.: Bull. Johns Hopkins Hosp. 31:225, 1920.

14. Moro, E.: Jahrb. f. Kinderh. 84:1, 1916.

15. Bessau, G., and Bossert, O.: Jahrb. f. Kinderh. 89:213, 269, 1919.

16. Plantenga, B. P. B.: Jahrb. f. Kinderh. 86:175, 1917. 
seemed useless to try to combat the carbon dioxid content only temporarily, and, secondly, the carbon dioxid content was frequently normal.

The food was breast milk or protein milk. In most cases no food seemed to be tolerated in any amount. In one case, a malt soup mixture seemed to do no harm. The fluid of the body was mostly maintained by means of subcutaneous and intraperitoneal injections. Transfusion was only done once so that we cannot report on its value. ${ }^{17}$

\section{CONCLUSIONS}

1. These cases seem to occur almost wholly in the summer months and are very rare in entirely breast fed children.

2. These cases are always accompanied by vomiting and diarrhea.

3. The etiologic factor or factors are as yet unexplained.

4. The carbon dioxid content and the nitrogenous constituents of the blood may be normal. Some of these cases may show only diminished carbon dioxid content. Others show a normal carbon dioxid content, but a markedly increased retention of the nitrogenous constituents, and, finally, some cases show both a diminished carbon dioxid content and a nitrogenous retention. Therefore, our studies would indicate that acidosis based on a lowered carbon dioxid content of the blood is not a constant or uniform finding.

The sugar and cholesterol content of the blood may be normal or high. There is apparently no relation between these constituents and either the nitrogenous or the carbon dioxid content.

5. In spite of all the variations in the chemical composition of the blood, there is no difference in the clinical picture or in the history of these cases.

17. Since the completion of this paper there has appeared ( $A m$. J. Dis. Child. 20:461 [Dec.] 1920) a very comprehensive paper by Marriott which deals, in part, with acute toxic nutritional disorders. His conclusions are that the toxic symptoms can be ascribed to a water loss from the body and a resulting concentration of the blood. This increased concentration of the blood in acute toxic conditions similar to the above has been confirmed by Rominger ( $Z$ tschr. f. Kinderh, 26:23, 1920) who also shows that this concentration persists for a long time during the reparative stage. 